\title{
Effekt einer additiven neurodynamischen Mobilisation im Vergleich zu einem Training der motorischen Kontrolle bei Patienten mit lumbaler Radikulopathie aufgrund eines Bandscheibenvorfalls: Eine randomisierte klinische Studie
}

\section{Effects of Adding a Neurodynamic Mobilization to Motor Control Training in Patients with Lumbar Radiculopathy due to Disc Herniation: A Randomized Clinical Trial}

Plaza-Manzano G, Cancela-Cilleruelo I, Fernández-de-las-Peñas C et al. Am J Phys Med Rehabil 2019; doi:10.1097/PHM.0000000000001295

\section{Zusammenfassung}

\section{Hintergrund}

Untere Rückenschmerzen betreffen einen Großteil der Bevölkerung. Sie beeinträchtigen häufig die Patienten und sind mit hohen Kosten für das Gesundheitssystem verbunden. Im Behandlungsmanagement dieser Patientengruppe gibt es moderate bis hohe Evidenzen für den Einsatz von motorischen Kontrollübungen bei chronischen unteren Rückenschmerzen [1]. Patienten mit lumbaler Radikulopathie stellen eine Untergruppe dar, bei denen die neurodynamische Mobilisation eine mögliche Behandlung ist. Die Literatur führt an, dass eine Reizung des Nervensystems möglicherweise mit einem Nervenödem, einer Ischämie und/oder einer Fibrose einhergeht, was zu Schmerzen und Funktionsstörungen führen kann [2, 3]. Der zugrundeliegende Mechanismus von neurodynamischer Mobilisation beinhaltet die Wiederherstellung der Homöostase im und um den Nerv sowie die Reduktion des intraneuralen Ödems durch den Einfluss auf die intraneurale Flüssigkeitsverteilung [4-6].

Diese Studie untersucht den Effekt einer neurodynamischen Mobilisation zusätzlich zu motorischen Kontrollübungen. Die Untersuchungsparameter sind Schmerz, die damit verbundene Beeinträchtigung, neuropathische Symptome, die Mechanosensitivität sowie die Druckschmerzschwelle bei Patienten mit einer lumbalen Radikulopathie aufgrund eines Bandscheibenvorfalls.

\section{Ziel}

Das Ziel dieser randomisierten klinischen Studie bestand darin, den Effekt von motorischen Kontrollübungen (MK) im Vergleich zur neurodynamischen (ND) Mobilisation mit MK bei Patienten mit lumbaler
Radikulopathie zu untersuchen. Es wurde die Hypothese aufgestellt, dass Patienten mit Radikulopathie bessere Ergebnisse mit der kombinierten Behandlung erzielen als allein mit der Behandlung mittels motorischer Kontrollübungen.

\section{Methode}

Die 32 Probanden mit unteren Rückenschmerzen, einem bestätigten Bandscheibenvorfall (BSV) und lumbaler Radikulopathie wurden nach dem Zufallsprinzip entweder zu acht Sitzungen mit motorischen Kontrollübungen plus ND-Mobilisation ( $n=16$ ) oder motorischen Kontrollübungen ohne ND-Mobilisation ( $n=16)$ zugeordnet. Die Untersuchungsparameter umfassten die Messung von Schmerz anhand einer nummerischen 11-PunkteSkala (NPRS), Beeinträchtigung mittels dem Roland and Morris Disability Questionnaire (RMDQ), neuropathische Symptome gemessen mit der Self-report Leeds Assessment of Neuropathic Symptoms and Signs Scale (S-LANSS), die Messung der Mechanosensitivität mittels Straightleg-raising-Test (SLR-Test) und die Druckschmerzschwelle gemessen mit einem Algometer.

Die Messungen wurden zu Studienbeginn, anschließend nach vier und acht Behandlungen sowie nach zwei Monaten durchgeführt. Beide Gruppen erhielten acht 30-minütige Behandlungseinheiten des Trainings der motorischen Kontrolle innerhalb von vier Wochen wie von Experten empfohlen [7]. In jeder Einheit korrigierte ein erfahrener Therapeut jeden Probanden individuell. Die Probanden wurden angewiesen, die Übungen jeden Tag für 20 Minuten insgesamt acht Wochen lang durchzuführen. Probanden der Kombinationsgruppe mit neurodynamischer Mobilisation bekamen zusätzlich eine Be- handlung mit einer Slider-Technik, jeweils fünf Minuten lang vor dem Training der motorischen Kontrolle. Die Unterschiede zwischen den Gruppen wurden anhand der Kovarianzanalyse ANCOVA berechnet. Zusätzlich wurden Effektstärken zwischen und innerhalb der Gruppen berechnet.

\section{Ergebnisse}

Es gab zu keinem Follow-up-Zeitpunkt signifikante Unterschiede zwischen den Gruppen in Bezug auf Schmerz (F: 1,269; p: 0,273; пр2: 0,043), Beeinträchtigung (F: 2,970; p: 0,101; пp2: 0,023) und Druckschmerzempfindlichkeit (N. tibialis: F: 0,582; p: 0,454; १p2: 0,026; N. fibularis communis: $F: 0,658$, p: 0,426 , пр2: $0,029)$. Beide Gruppen erzielten in diesen Parametern ähnliche Verbesserungen. Die Effektgröße zeigte in diesen Parametern kleine Unterschiede zwischen den Gruppen und große Unterschiede innerhalb der Gruppen. Zwei Parameter zeigten einen signifikanten Unterschied zwischen den Gruppen. Bei den Patienten, die eine kombinierte Behandlung der MK- und ND-Mobilisation erhalten hatten, verbesserten sich signifikant die Parameter der neuropathischen Symptome (F: 8,559; p: 0,008; пр2: 0,373) mit großer Effektstärke zwischen den Gruppen und bezüglich der Mechanosensitivität (F: 7,512; p: 0,013; пр2: 0,220) mit moderater Effektstärke zwischen den Gruppen.

\section{Schlussfolgerungen}

Die Behandlung mit ND-Techniken additiv zu einem Training der MK führte zu einer Verringerung der neuropathischen Symptome (S-LANSS) und einer Reduktion der Mechanosensitivität (SLR-Test), es zeigten sich jedoch keine signifikanten Änderungen in Bezug auf Schmerz, Beeinträchtigung und Druckschmerzempfindlichkeit im Vergleich zum Training der motori- 
schen Kontrolle alleine. Weitere Studien sind erforderlich, da die Unterschiede zwischen den Gruppen keine klinische Relevanz erlangten.

\section{Kommentar}

Die Autoren untersuchten, ob eine kombinierte Behandlung bei einer Patientengruppe mit lumbalen Radikulopathien durch MKÜbungen und ND-Mobilisation einen Vorteil im Vergleich zu MK-Übungen ohne ND-Mobilisation zeigt. Es zeigte sich eine signifikante Verbesserung der neuropathischen Symptome (S-LANSS) und eine Reduktion der Mechanosensitivität (SLR-Test). Alle anderen Parameter (Schmerzintensität, Druckschmerzempfindlichkeit und Beeinträchtigung) verbesserten sich gleichermaßen in beiden Gruppen. Durch die fehlende Kontrollgruppe bleibt unklar, in welchem Maß sich die Kontrollgruppe im Vergleich verbessert hätte und ob sich die Interventionsgruppen signifikant und klinisch relevant dazu verbessert hätten.

Die Auswahl der Behandlung der Probanden in beiden Gruppen mit motorischen Kontrollübungen ist nicht ganz nachvollziehbar, denn hier wird ein Review zitiert, das auf die Behandlung von unspezifischen chronischen Rückenschmerzen abzielt [1]. Radikulopathie ist eine spezifische Diagnose, die Autoren geben jedoch nicht an, wie sie eine Radikulopathie definieren. Radikulopathie ist im Gegensatz zu radikulären Schmerzen mit neurologischen Defiziten (loss of function) definiert $[8,9]$. Diese Minuszeichen werden nicht unter den Einschlusskriterien genannt. Einschlusskriterien der Studie waren ein im MRT sichtbarer Bandscheibenvorfall zwischen L4 und S1, ausstrahlende Symptome bis in den Fuß, Schmerzen seit mindestens drei Monaten, vermehrte Schmerzen durch Husten, Niesen oder Belastung und ein positiver SLR zwischen $40^{\circ}$ und $70^{\circ}$. Diese Einschlusskriterien umfassen somit diverse Schmerztypen wie somatisch übertragene Schmerzen aus Gelenken, Muskeln etc., erhöhte Mechanosensitivität und radikuläre Schmerzen, die nozizeptive und neuropathische Schmerzkomponenten beinhalten können. Für eine Radikulopathie fehlen bei den Einschlusskriterien die konkrete Auflistung der Minuszeichen, denn ein durch MRT bestätigter BSV kann auch symptomfrei sein [10].

Das Ergebnis dieser Studie zeigt jedoch, dass eine subgruppenspezifische Behandlung funktionieren könnte. Wenn die Gruppe, die hier untersucht wurde, eine Gruppe mit erhöhter Mechanosensitivität teilweise mit nozizeptivem oder radikulärem Schmerz, aber ohne konkrete Minuszeichen war, wäre erklärbar, warum sich gerade diese Parameter signifikant verbesserten. Diese Erklärung würde zu den Ergebnissen einer anderen Studie passen, die sich mit der subgruppenspezifischen Behandlung beschäftigte und beobachten konnte, dass sich die Symptome der behandelten Gruppe mit erhöhter Mechanosensitivität in höherem Maß durch eine ND-Mobilisation verbesserten als die der Probanden aus einer anderen klassifizierten Gruppe [11].

Darüber hinaus sind die Aspekte Dosierung und Prognose interessant. Die Autoren zeigten, dass sich die Symptome der Probanden im SLR-Test nach acht Behandlungen im Gegensatz zu vier Behandlungen deutlich verbesserten. In den abgebildeten Grafiken ist zu sehen, dass bis zur vierten Behandlung nur wenige Effekte sichtbar wurden. Auf die klinische Praxis übertragen bedeutet das, dass bei Patienten mit einer erhöhten Mechanosensitivität mit unteren Rückenschmerzen und Ausstrahlungen in das Bein erst nach der vierten Behandlung mit MKund ND-Mobilisation erste Effekte und deutliche Effekte erst nach der achten Behandlung erwartet werden dürfen.

\section{Autorinnen/Autoren}

Camilla Kapitza, PT BSc, MSc Phys, PT OMPT-dvmt

INAP/0 Institut für angewandte Physiotherapie und Osteopathie

c.kapitza@hs-osnabrueck.de
Literatur

[1] Saragiotto BT, Maher CG, Yamato TP et al. Motor control exercise for chronic nonspecific low-back pain. Cochrane Database Syst Rev 2016; 1: CD012004Sociaty fp

[2] Butler DS. The Sensitive Nervous System. Adelaide, Australia: Noigroup Publications; 2000

[3] Shacklock MO. Neurodynamics. Physiotherapy $1995 ; 81: 9-16$

[4] Gilbert KK, Roger James C, Apte G et al. Effects of simulated neural mobilization on fluid movement in cadaveric peripheral nerve sections: implications for the treatment of neuropathic pain and dysfunction. J Man Manip Ther 2015; 23: 219-225

[5] Brown CL, Gilbert KK, Brismee JM et al. The effects of neurodynamic mobilization on fluid dispersion within the tibial nerve at the ankle: an unembalmed cadaveric study. J Man Manip Ther 2011; 19: 26-34

[6] Boudier-Revéret M, Gilbert KK, Allégue DR et al. Effect of neurodynamic mobilization on fluid dispersion in median nerve at the level of the carpal tunnel: a cadaveric study. Musculoskelet Sci Pract 2017; 31: 45-51

[7] Costa LO, Maher CG, Latimer J et al. Motor control exercise for chronic low back pain: a randomized placebo-controlled trial. Phys Ther 2009; 89: 1275-1286

[8] Bogduk N. On the definitions and physiology of back pain, referred pain, and radicular pain. Pain 2009; 147 (1): 17-19

[9] IASP TFoT. Part III: Pain terms, a current list with definitions and notes on usage. Seattle: IASP Press; 1994

[10] Schmid AB, Tampin B. Section 10, Chapter 10: Spinally Referred Back and Leg Pain. In: International Sociaty for the Study of the Lumbar Spine, Hrsg. Lubar Spine Online Textbook. www.wheelessonline.com/ISSLS/ section-10-chapter-10-spinally-referredback-and-leg-pain/ (13.12.2019)

[11] Schäfer A, Hall T, Müller G et al. Outcomes differ between subgroups of patients with low back and leg pain following neural manual therapy: a prospective cohort study. Eur Spine J 2011; 20 (3): 482-490. doi: 10.1007| s00586-010-1632-2

\section{Bibliografie}

DOI https://doi.org/10.1055/a-1086-7405 physioscience 2020; 16: 42-43 (c) Georg Thieme Verlag KG, Stuttgart · New York ISSN 1860-3092 